\title{
Torsion of huge epididymal cyst in a 16-year-old boy: case report and review of the literature
}

\author{
Cosimo Bleve, Maria Luisa Conighi, Valeria Bucci, Lorenzo Costa, Salvatore Fabio Chiarenza \\ Department of Pediatric Surgery and Pediatric Minimally Invasive Surgery and New Technologies, San Bortolo Hospital, \\ Vicenza, Italy
}

\begin{abstract}
Epididymal cysts (ECs) are relatively common in adults, rare in children. Normally their treatment is conservative. They may be situated anywhere in the organ, frequently in the region of the head. Torsion of these cysts is extremely rare in both children and adults, causing acute scrotal swelling. The diagnosis is intraoperative. A 16-year-old boy was referred to our Divisional Clinic by the treating physician for scrotal swelling appeared 4 months earlier. Absence of a history of minor scrotal trauma. Ultrasonography showed a $40 \times 50 \mathrm{~mm}$ fluid-filled right para-testicular mass. We performed surgery finding a large black cyst connected to the head of the epididymis with $720^{\circ}$-degrees rotation. Histology revealed an acquired EC. The particularity of our case is due to the absence of symptoms in association with a big EC twisted of $720^{\circ}$ degrees. This is the only case reported in literature. All patients with EC torsion reported presented symptoms related to acute scrotum.
\end{abstract}

Correspondence: Cosimo Bleve, Department of Pediatric Surgery and Pediatric Minimally Invasive Surgery and New Technologies, San Bortolo Hospital, Viale Rodolfi 37, Vicenza, Italy.

Tel.: +39.0444.752642 - Fax: +39.0444.752643.

E-mail: cosimo.bleve@aulss8.veneto.it

Key words: Cyst; Epididymis; Scrotal masses; Torsion.

Contributions: $\mathrm{CB}$, MLC prepared the initial draft of the manuscript and revised all subsequent versions. $\mathrm{CB}$ reviewed the literature data; $\mathrm{LC}$ and VB provided care and follow-up data for the case; CB and SFC reviewed the early versions of the manuscript and proposed the key message of the manuscript providing critical input to the revisions of all versions of the manuscript. All authors read and approved the final manuscript.

Conflict of interest: the authors declare no potential conflict of interest.

Funding: none.

Received for publication: 17 July 2017

Accepted for publication: 13 March 2018.

This work is licensed under a Creative Commons Attribution NonCommercial 4.0 License (CC BY-NC 4.0).

(C) Copyright C. Bleve et al., 2018

Licensee PAGEPress, Italy

La Pediatria Medica e Chirurgica 2018; 40:162

doi:10.4081/pmc.2018.162

\section{Introduction}

Epididymal cysts (ECs) is a benign mass that usually develop in adult men, but it is rare in children, with a prevalence of 5-20\% according to varying series reported in the literature. ${ }^{1}$ They present as single or multiple, uni- or bi-lateral spherical cysts localized most frequently in the head of the epididymis. Although the cause of EC and spermatocele is often unknown, it may be caused by epididymal ducts obstruction. They are usually of lymphatic origin. When small, ECs remain undetected and can be found in approximately $30 \%$ of asymptomatic patients having scrotal ultrasound for other reasons. On the contrary to adolescent in adults most of these cysts are spermatocele. ${ }^{2}$ With a certain diagnosis (ultrasound), their treatment is conservative under elective condition. Rarely, as result of trauma or torsion of these cysts, exploration of the scrotum is required to rule out other pathologies such as testicular torsion. An EC torsion is extremely rare especially in young boys and to the best of our knowledge only 6 cases have been reported in literature. ${ }^{3}$

\section{Case Report}

The treating physician has referred a 16-year-old boy to our Service for right scrotal swelling appeared 4 months before. The patient reported the absence of a history of minor scrotal trauma. He had no fever or urinary symptoms. Physical examination demonstrated left scrotal region and left testis normal. On the right side, tenderness and swelling of the right hemiscrotum; the testis was appreciated of regular consistency, with vague margins, located on the inferior-posterior area. Superiorly was appreciated an elastic area as loculated hydrocele. No pain on palpation. At the lower pole of the testis was appreciable a hard consistency area, $1 \mathrm{~cm}$ in length, painless. Abdominal examination was unremarkable. The inguinal canal was not engaged. Routine hematology, serum biochemistry, and urine analysis were normal. Color-Doppler-Ultrasonography showed both testes with normal parenchymal architecture and echogenicity with no perfusion defect as the left epididymis. On the right side an echo-free cystic structure of $4,4 \mathrm{~cm}$ of diameter within the head of the epididymis with no perfusion (Figure 1). The patient underwent to programmed surgery (we have not signs of acute pathology or suffering testis) after 1 week: scrotal exploration under general anesthesia. After opening the tunica vaginalis, we found a large cystic mass $(5 \times 4 \mathrm{~cm})$ connected to the head of epididymis by a pedicle twisted for $720^{\circ}$ degrees. The testis instead was normal (Figure 2). The epididymis was edematous. The cyst was untwisted and excised maintaining the integrity of the 
epididymis (Figure 3). On opening, the cyst contained semi-clear fluid containing spermatozoa in microscopic examination. The cyst presented a thin wall with congested blood vessels with angiectasie and serous content. The patient was discharged one day after surgery and physical examination was normal during 12 months' follow-up.

\section{Discussion}

ECs usually develop in adult men. Although uncommon in children, they occur in a number of boys in a pubertal age. Usually most of ECs in children involute with time and therefore do not require treatment. If become symptomatic and/or doesn't resolve or suddenly starts increasing in size, should be treated. ${ }^{2}$ Acute scrotum in these cysts (i.e., torsion of EC or spermatocele) is extremely rare.

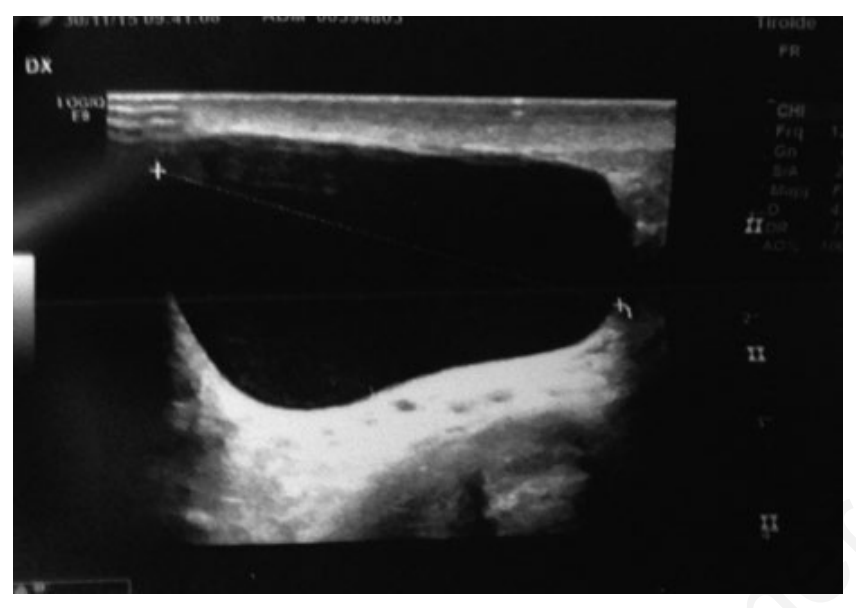

Figure 1. Echo-free cystic structure of $4,4 \mathrm{~cm}$ of diameter within the head of the epididymis with no perfusion.
With a sudden and acute onset of symptoms and the presence of tenderness in physical examination that is similar to testicular torsion, exploration of the scrotum is indicated.1-3 In this case, the possible etiologies, causes of acute scrotum are different: testicular torsion, epididymorchitis, torsion of appendix testis, trauma, and strangulated inguino-scrotal hernia. Tumor, idiopathic scrotal edema and Henoch-Schönlein purpura are other possible causes.

The incidence of ECs in children ranges from 5\% to 20\%. Their nature (degenerative or result of endocrine-disrupting agents acting

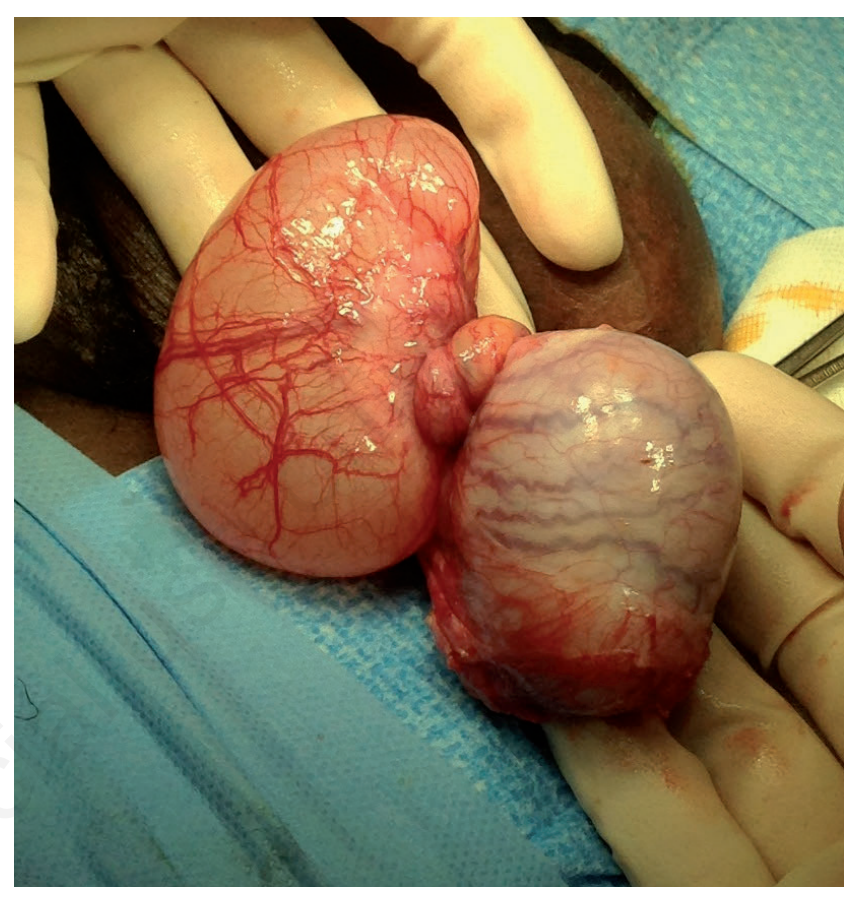

Figure 2. Large cystic mass $(5 \times 4 \mathrm{~cm})$ of the head of epididymis twisted for $720^{\circ}$ degrees with normal testis.

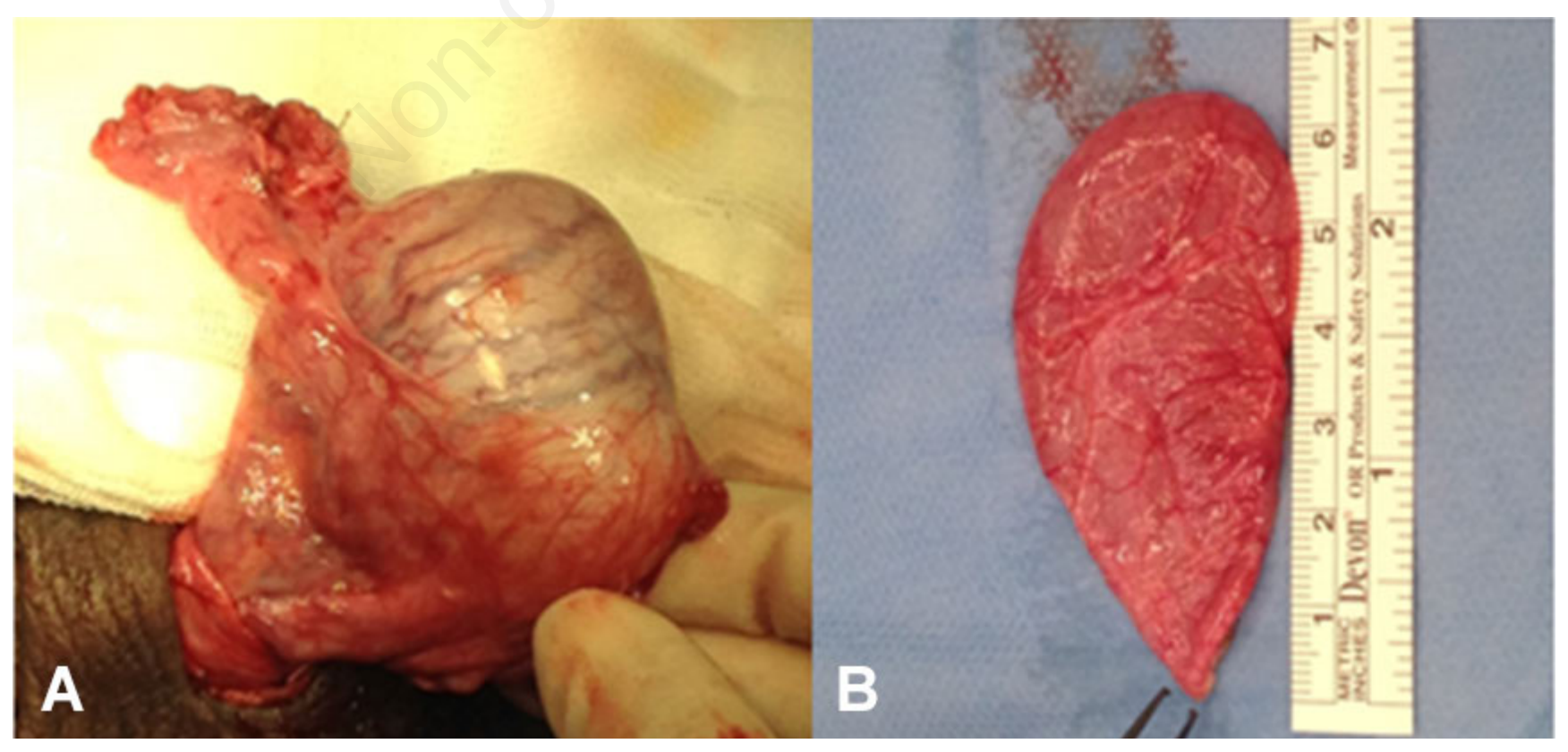

Figure 3. A) Excision of the cyst preserving the integrity of the epididymis; B) Excised cyst. 
fetally or postnatally) and their functional significance are unknown with certainty. ${ }^{1}$ Usually they are acquired rather than congenital, which is why they typically occur in older men; usually they are benign. The cysts are usually painless and, because most of these cysts are asymptomatic, treatment is conservative. Swelling is usually located in the upper part of the testicle because the epididymis lies behind the testis. Hedelin 4 described a torsion of a mass in the epididymal head. By a revision of literature, torsion of ECs is extremely rare condition and only six cases has been reported, all characterized by acute symptoms associated. ${ }^{1}$

With our patient, we did not have history of scrotal trauma or other urinary symptoms. We appreciated tenderness and swelling of the right hemiscrotum with an elastic area as loculated hydrocele on the upper pole of the testis and a hard consistency area, $1 \mathrm{~cm}$ in length, inferiorly without pain on palpation.

In the majority of patients with EC, conservative management has been suggested although there is not consensus about the most adequate therapy of pubertal ECs. The majority of these cysts involute with time.

If become symptomatic, do not resolve or suddenly start increasing in size, should be treated surgically or by sclerotherapy. As reported by Niedzielski et al., ${ }^{2}$ we don't approve and recommend sclerotherapy in the developing gonads of boys and adolescents. There are not reports about effectiveness of this procedure while diagnostic mistakes about nature of suspected ECs are reported in literature.

Indeed, there are cystic tumors which can present like EC on scrotal ultrasound like adenomatoid tumor of epididymis, 5 epidermoid cyst (monodermal teratoma) of the testis. According to Heidenreich and Reinberg, ${ }^{6}$ the ultrasonographic appearance of epidermoid cyst of the testis is not specific and exploratory surgery is recommended in all cases. Although extremely rare, these possible etiologies must be considered.

\section{Conclusions}

According to our experience and opinions, as confirmed by literature, we established criteria for surgical treatment of ECs. Usually small asymptomatic cysts (under $10 \mathrm{~mm}$ ) are followed with clinical and sonographic follow-up; with cyst between 10 and 20 $\mathrm{mm}$ conservative management is followed if they are asymptomatic; with symptoms they should be excised. Big and symptomatic/ asymptomatic ones must underwent surgical excision.

The particularity of our case is due to the absence of symptoms in association with a big EC twisted of $720^{\circ}$ degrees. This is the only case reported in literature. All patients with torsion of EC reported presented with symptoms related to acute scrotum, namely scrotal swelling and pain. Some cases of testicular torsion in association with ECs are described; for that reason, in our opinion is important to educate the patients with EC treated conservatively for warning signs, which are similar to testicular torsion.

\section{References}

1. Erikçi V, Hoşgör M, Yıldız, et al. Torsion of an epididymal cyst: a case report and review of the literature. Turk J Pediatr 2013;55:659-61.

2. Niedzielski J, Miodek M, Krakós M. Epididymal cysts in childhood-conservative or surgical approach? Pol Przegl Chir 2012;84:406-10.

3. Ameli M, Boroumand-Noughabi S, Gholami-Mahtaj L. A 14year-old boy with torsion of the epididymal cyst. Case Rep Urol 2015;2015:731987.

4. Hedelin H, Eddelan A, Johansson S, Mark J. Torsion of a large epididymal mass. Br J Urol 1986;58:560-1.

5. Vick CW 3rd, Klein FA, Schneider V. Adenomatoid tumor of epididymis simulating benign cyst on scrotal ultrasound. Urology 1991;38:369-71.

6. Reinberg Y, Manivel JC, Llerena J, et al. Epidermoid Cyst (monodermal teratoma) of the testis. Br J Urol 1990;66:648-51. 